\title{
Surface Texturing with Laser Micro Cladding to Improve Tribological Properties.
}

\author{
Nerea OTERO ${ }^{* 1}$, Pablo ROMERO $^{* 1}$, Alejandro GONZALEZ ${ }^{*_{1}}$, Alessandra SCANO $^{*_{2}}$ \\ ${ }^{*}$ AIMEN Technology Centre, Laser Applications Centre (CAL); c/ Relva 27A - Torneiros; 36410 \\ Porriño (Pontevedra), Spain \\ E-mail: notero@aimen.es \\ *2 AIN, c/ San Cosme y San Damián s/n; 31191 Cordovilla-Pamplona, Spain.
}

\begin{abstract}
In this work, laser microcadding with continuous wave laser is proposed as a productive, flexible and simple method for surface modification of 3D metallic components, to generate microfeatures which influence the surface interaction of tribological pairs and surfaces under lubricated contact conditions. In this paper, texturing additive process by means of laser micro-cladding [L $\mu \mathrm{C}]$ is presented. $\mathrm{L} \mu \mathrm{C}$ texturing has been developed for generation of functional and tribological surfaces on metallic components. On one hand, cladding allows the engineering of surface properties, as a wide range of clad materials can be chosen for increasing properties and performance of the components. On the other hand, microtexturing adds the possibility of generating controlled geometrical features in the micron scale, which influence the interactions with the lubrication fluids and the wearing mechanisms. The proposed $\mathrm{L} \mu \mathrm{C}$ technique combines these two advantages in a single step for a novel surface treatment. DOI:10.2961/jlmn.2012.02.0005
\end{abstract}

Keywords: Laser micro-cladding, Surface modification, Selective laser melting, Metallic powder, Single-mode fiber laser.

\section{Motivation}

Engineered components are normally designed to obtain a compromise between shape, material, cost and performance; only general surface features like roughness are usually taken into consideration. But surface is where the physical interaction with the medium takes place for all engineered components and it is the source for most process inefficiencies, like heat losses or friction.

In case of lubricated pairs (most mechanisms, motors and generators), surface metal-metal contact, and interaction with viscous fluids causes the largest portion of energy loss and efficiency drop.

The notches generated by surface particles higher than the lubricant film are detrimental to the life of components subjected to conformal friction, by accelerating cracking and fatigue phenomena. However, controlled surface texturing has demonstrated its ability to extend the life of components under friction. This apparent contradiction can be explained through the modification of the fluid dynamic of lubricated contact when these textures are present.

The mechanism by which the texture extends the life of lubricated friction pairs is by improving the stability and integrity of the lubricant film, preventing or reducing the metal-metal contacts and the effects of contaminants on the component surface [1-4].

\subsection{Requisites}

The main requirement for the structures to be made is that they must withstand long term operation in moving components in contact with lubricants and harsh environments without degradation of their geometrical quality. The beneficial effect of surface microstructures largely depends on the size and distribution of microfeatures on the surface, and a modification of feature size can even turn into detrimental to the performance of the component. That is why a high dimensional stability in time is mandatory for exploiting the energy-saving potential of the microfeatures.

All these requirements can be translated to high wear resistance of the clad track, as well as high adhesion to parent material. In most cases, chemical and mechanical requisites demand that the chemical composition of the cladded track need to be the same than the one in the original powder, thus low dilution is required. When surfacing is made by fusion, this implies strong requirements on process-induced defects, stresses and thermal impact on parent material.

\section{Microcladding technology}

There are many surface modification techniques industrially available, from additive (coating) to subtractive (machining) techniques. These techniques, either do not provide the geometrical precision required for tribological improvement, or are far too slow to provide reasonable productivity.

Techniques such as mechanical transferring or embossing of structures on the metallic surfaces, electro discharge machining and similar, are productive and have high precision, but lack of flexibility or adaptability to the macrogeometry of the workpiece.

The preferred actual way to produce microfeatures on aerostructures is the use of rubber coatings containing the micro-geometries. This, nevertheless, is not suitable if the designer needs to keep the surface properties of the work- 
piece materials for heat transfer, wear or chemical compatibility.

Laser micromachining is intended to overcome all these limitations, but available pulsed lasers make it quite slow and expensive by the moment.

Additive manufacturing allow improving surface functionality by introducing a new material in the interface with the medium. Laser cladding (several millimeters in track width and height) has been largely reported as a high quality surfacing process due to the ability to reach small dilutions, controlled heat effects and low process induced stress. Laser cladding is usual in aerospace repairing, chemical and nuclear industry, etc.

Laser microcladding is the technique of surfacing by melting of material on the substrate, when the produced features are micron-sized. The need for producing features of a few tens of microns implies certain stringent requirements on the laser sources, powder feeding equipment and powder characteristics. Microcladding has been developed [5-6] for micro-features production, precision repairing and direct manufacturing.

Microcladding is still far from industrial applications, but it deserves large attention due its potential to provide a cheap and fast way to produce certain texturing of functional surfaces.

The main limitation and technical complexity of the process is imposed by the powder size required to produce features under 80 microns. As well, the other main requirement of the technique arises in the beam quality of the laser source and its spot size. It is normally required that the powder size is at most, half the size of the smaller feature to be produced. Similar constrains can be imposed to laser beam size. The process works better when the powder size is under the beam size. Metallic powders under 30 microns have high cohesive behaviour which prevents it from easy flowing. Special fluidizing methods are needed to overcome their cohesive forces, like superpulsation of gas flow, vibration, ultrasonics, additives, etc...This make the process setup more complex and expensive.

For this work, desired feature size is in the order of tens of microns. This condition implies stringent requirements on the laser source, powder feeding equipment and feed material characteristics.

The main technological limit using powders with particle size fewer than $50 \mu \mathrm{m}$, is the transport of the powder to the workpiece. Pneumatic transport of powders with high surface/mass ratio is extremely difficult, due to their trend to agglomerate and break the fluid bed. Metallic powder under 50 microns lays in all cases in the " $C$ " class of powder according to Geldart classification [7], which is considered not suitable for pneumatic transportation, requiring high density gas, electrostatic charging, conditioning additives, or additional energy source: ultrasounds, acoustic pressure or magnetic pulses [8]. In this work an alternative approach is proposed and developed: powder preplacement.

\section{Experimental Work}

\subsection{Equipment}

The laser source used in this work is a single mode fibre laser (Trufibre400 from Trumpf), with a fibre core diameter of 11 microns and a maximum $\mathrm{CW}$ power of $400 \mathrm{~W}$.
This equipment is representative of the new generation of high brilliance industrial laser sources, able to produce high densities for fast and accurate processing.

Most shapes were produced by using the laser in $\mathrm{CW}$ mode and using the controller of the beam stirring system to control the laser gate. Only complex geometries were produced by using the laser in modulated mode.

All shapes are produced using galvoscanner optics to guide the laser radiation to the workpiece. The theta field lenses provide a working field of $36 \mathrm{~cm}^{2}$ with fixed focus distance of $135 \mathrm{~mm}$. The optical magnification of this system is 1.5 , which yields a theory focal point of 17 microns. This optical arrangement takes benefit from a large Rayleigh length of several millimeters, at least two orders of magnitude larger than the spot size.

\subsection{Materials}

Different materials were used in $\mathrm{L} \mu \mathrm{C}$ generation of micro-features, in order to test the potential of the technology, as well as the dependence of results on the nature of the parent and added material. Tests were made on flat, polished samples taken out from different base materials.

Tested materials had been selected according to the potential applications of the developed technique, with the improvement of tribological behaviour as main objective.

Studied base materials include different steels, mainly tool steels and titanium. Different commercial metallic powders were used during the trials: H13 steel (high $\mathrm{Cr}$ ), H20 steel (high W), titanium Grade-V, nickel, Co-Cr alloy. Very low dilution is mandatory to avoid metallurgical problems.

A cold working grade D-2 steel, with high $\mathrm{C}$ and $\mathrm{Cr}$ content, was used as base material. This steel was selected in base of it excellent wear. This material is commonly employed for forging, die casting and drawing dies. D2steel is used to test the ability of the method for improving lubrication, by means of producing structures which improve lubricant pressure and film thickness.

Titanium Grade-V (Ti6Al4V) is not a good choice for friction applications, but in several aerospace components, such pieces of turbines, its use can be mandatory without any coating or chemical modification, in order to maintain other important properties inherent to titanium. The base material has biphasic microstructure which needs to be kept after the process to maintain its mechanical and corrosion resistant properties.

Using titanium powders as additive material, final performance of modified surface will be better for friction applications. Ti powder under $20 \mu \mathrm{m}$ in size was used in this work.

Co-Cr-W alloy powder was used for improved wear resistance and good metallurgic compatibility $(\mathrm{D}<20 \mu \mathrm{m})$, as well as inconel $(50 \mu \mathrm{m}<\mathrm{D}<20 \mu \mathrm{m})$ Some special mixtures, like Stellite 6 with TiC nanoparticles attached to the grains were also used by means of high energy milling, to provide better performance thanks of nanoparticle dispersion in cladding track.

\subsection{Experimental procedure}

A pre-placed powder consolidation method is proposed for the production of cladding tracks under $50 \mu \mathrm{m}$ in thick- 
ness, thanks to the high beam quality of a diffractionlimited solid state laser source for performing the powder consolidation.

A thin layer of powder is placed on the surface to be textured. Powder pre-placement can be performed as a different step prior to microstructuring, or can be automatically performed simultaneously to the laser consolidation, in order to improve the productivity of the process.

Powder pre-placement was performed using simple techniques based on painting technology, or on the application of colloidal suspension, with the objective of avoid the need of fluidizing and transporting the particles. Two different pre-placement methods were tested, both taking advantage of the cohesive nature of the powder to get a homogeneus layer: in the dry placement, the powder is directly deposited on the workpiece and a scrapper blade is used to get the even distribution. The resulting layer height depends on the distance of the blade to the surface. The homogeneity and density of the predeposited layer depends on the particle geometry and size distribution of the powder.

In wet placement, a liquid suspension of the powder is first prepared by agitation or ultrasonic vibration of the powder within a solvent, normally isopropyl alcohol. The resulting suspension, in the form of dense and fluid slurry, can be applied by means of spraying or by painting the surface. By wet method, powder placing is easier, especially in 3D surfaces. In this case, the thickness of the powder later depends on many governing factors, like fluid-solid interaction, suspension concentration, application method, etc.

The deposited powder is consolidated selectively and locally by means of a scanned laser source, with enough energy to melt both the powder and a small volume of the substrate, to improve their adhesion, but with low dilution.

\section{Results and discussion}

Produced tracks and structures by powder consolidation, had been characterized to assess their geometrical and mechanical quality, as well as the expected performance. Characterization techniques include interferometric surface and contact microprofiling, metallographic study, SEM and wear tests.

\subsection{Parametric Analysis and Geometrical Quality of Microstructured Surfaces.}

For each combination of base and clad material, a test matrix was performed combining variations of the different governing parameters of the process, such as scanning speed, laser power and focus position. Performed tracks are hence characterized by using different criteria, for selection of the most adequate combination of parameters:

- Geometrical quality and characteristics

- Metallurgical quality: dilution, defectology, heat affected zone

As expected, metallographic observations revealed a strong dependence of the microcladding track width with the size of the powder particles, as well as the spot size of the laser radiation. The depth of the molten and heat affected zone is directly connected with the power density of the laser radiation and inversely proportional to the scanning speed on the surface, so it can be related with the total en- ergy density, as happens in the case of deep penetration welding.

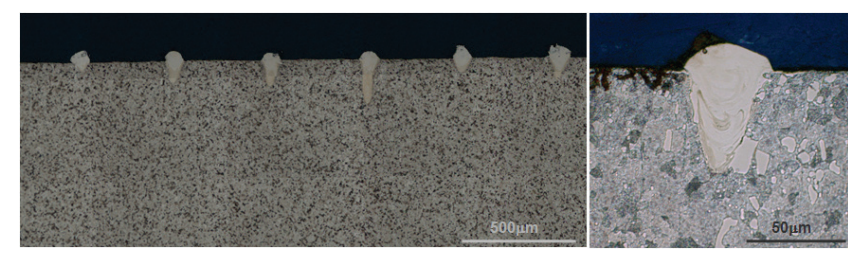

Fig.1 Series of tracks with different combinations of process parameters (H20 powder on D2). Detail of one track.

This sequence was repeated for all combinations of materials and powder pre-placing conditions, with and without gas shielding, resulting in a large amount of test cladding tracks, which could be compared in terms of penetration and dilution.
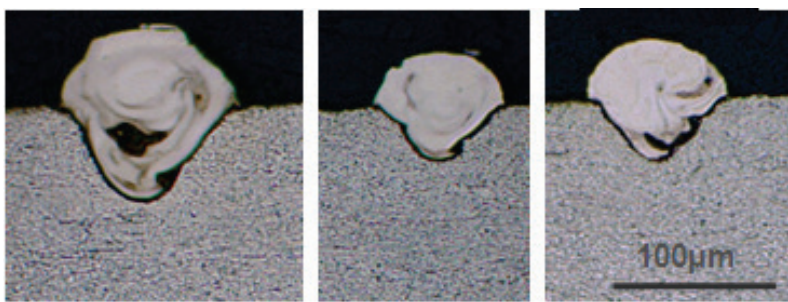

Fig. 2 Micrographs of tracks with different combinations of parameters (Ni alloy 718 on Ti Grade-V).

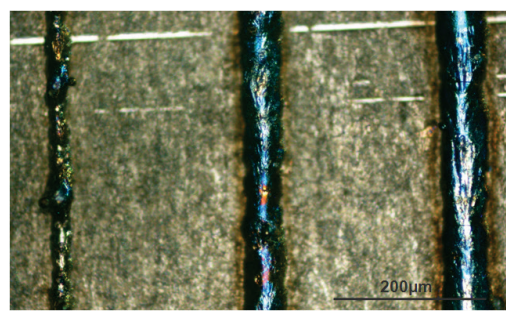

Fig. 3 Tracks with different combinations of parameters (Ni alloy 718 on Ti Grade-V).

In the previous figures, details of the microtracks can be seen with their transversal cuts, showing very variable results depending on the power density applied, from unstable powder consolidation at lower fluences, to excessive melt penetration. A valid parameter window can be selected for each material, which in all cases results in fluences of about $10 \mathrm{MW} / \mathrm{cm}^{2}$.

Keeping the spot size constant at 22 microns just on the powder surface, the following results arise from the analysis of cladding tracks:

For most materials combinations, a window of power between 20 and $50 \mathrm{~W}$ gives good results with low HAZ, when combined with high quality powder (monomodal distribution of spherical powder under 20 microns) and gas shielding.

Depth and width are kept stable when power is kept under $50 \mathrm{~W}$ and speed over $600 \mathrm{~mm} / \mathrm{s}$. Final result is more dependent on laser powder than on scanning speed [9]. At very low power (20W and bellow) the process becomes unstable, and even the melting stops, yielding irregular or discontinuous tracks with low adhesion to the parent material. 
After comparing the different conditions for each material, the best combination of operation conditions was chosen to perform several parallel tracks to form a riblet-like structure and asses the stability of the method.

\begin{tabular}{|l|l|l|l|}
$\begin{array}{c}\text { Base } \\
\text { Material }\end{array}$ & \multicolumn{1}{c|}{ Powder } & \multicolumn{1}{c|}{$\begin{array}{c}\text { Power } \\
(\mathbf{W})\end{array}$} & $\begin{array}{c}\text { Scanning Speed } \\
(\mathbf{m m} / \mathbf{s})\end{array}$ \\
\hline Ti & $\mathrm{Ti}(<20 \mu \mathrm{m})$ & 25 & 700 \\
\hline Ti & $\mathrm{Ni}(<50 \mu \mathrm{m})$ & 50 & 700 \\
\hline Ti & $\mathrm{Cr} / \mathrm{Co}(<50 \mu \mathrm{m})$ & 50 & 700 \\
\hline D2 & $\mathrm{H} 20(<20 \mu \mathrm{m})$ & 50 & 800 \\
\hline
\end{tabular}

Fig. 4 Selected parameters for different combination of materials.

Microcladding track geometry was characterized by using a optical profiler, which performs non-contact 3D surface profilometry and surface roughness characterization. This procedure was used to take 3D topographical data of the microtextured surfaces and obtain quality indicators for the different microcladding tracks.

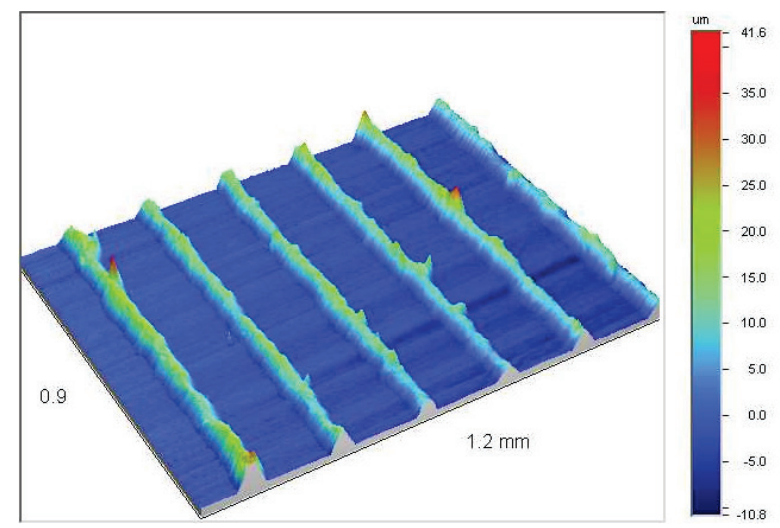

Fig. 5 3D topographical data of a sample of tool steel.

As previously said, suspension preparation and powder quality strongly influence the geometry. In some samples, spots of excessive size (humps) are detected, due to powder placement defects (layer non homogeneous), because of the different distribution size of powder particles.
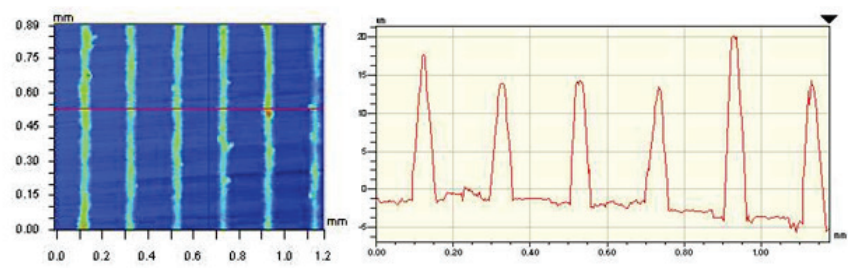

Fig. 6 Transversal profile of a riblet-like structure on tool steel.

The general aspect of the geometry is very good, with stable track thickness of 50 microns easily attainable, and a very stable mean height and roughness for the tracks, of about 30 microns in height.

\subsection{Geometrical limits of the method}

For wear resistant applications, biofouling resistant surfaces and rotating components (conformal contacts in motors and turbines), special geometries can be required as alternative to standard parallel riblets, which can require presence of track overlaps or curved features.
In order to define the geometrical limits of the method, some cross lines were generated. In this case, the selected material was titanium grade $\mathrm{V}$ as both base material and clad material. The reason of this selection was the objective of increase the wear resistance of titanium, by means of texturing with the same material to maintain all the properties of base material.

The best combination of parameters selected for titanium as both base and clad materials, was used to develop the study of two different effects: minimum possible intersection angle to generate stable and homogeneous cross tracks, and the effect of different distance between parallel tracks. The purpose of the experiment was to test the behaviour of the tracks in cases where the prior existence of a string can alter the process. In the case of crossed tracks studied at different angles, different features in the intersecting track formation were identified, included dragging (produced by surface tension effect in the contact with the prior solidified track) and humping due to remelting and displacement of the material of the previous track. The size of such features depends on the length and angle at which the crossing happened. With crossing angles under $10^{\circ}$ it is not possible to obtain homogeneous tracks, mainly due to said dragging effect resulting in defective profiles.

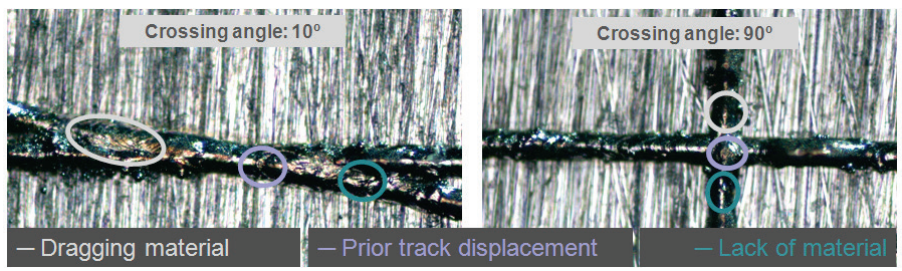

Fig. 7 Transversal profile of a riblet-like structure on tool steel.

In the case of distance study, different effects were observed, namely in distances under $90 \%$ of the track width. Depending on the distance, the features can be defined as: two well defined tracks, lack of material in the first track (remelting effect) and union to prior track (undiferenced tracks). Track distance under 10 microns causes non homogeneous tracks and with lack of material.
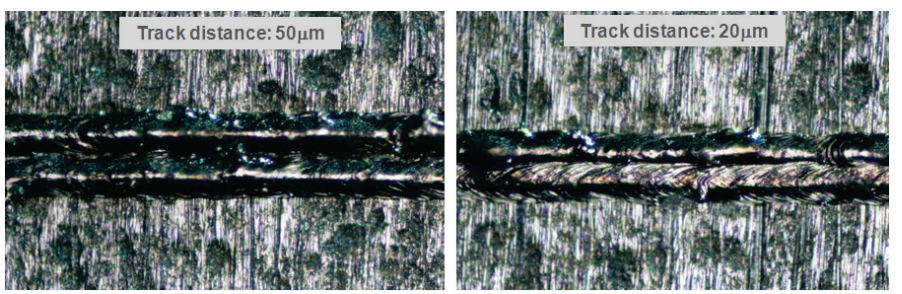

Fig. 8 Transversal profile of a riblet-like structure on tool steel.

\subsection{Complex geometries}

Once the limits of the technique were defined and the evidence of texture generation with close and crossing tracks is assessed, the ability to generate complex geometries was studied.

Circular geometries were generated, different distance between radial tracks and crossing lines, as well as the generation of biomimicking textures. These kinds of geometries are suitable for certain particular friction pair configurations, such as rotating components working in conformal 
contact, or friction with presence of lubrication in 3D forming tools (drawing, stamping, ...).

The main features of the structures are maintained (their spanning between 50 and 250 microns, track width of around 50 microns and height of a few tens of microns).
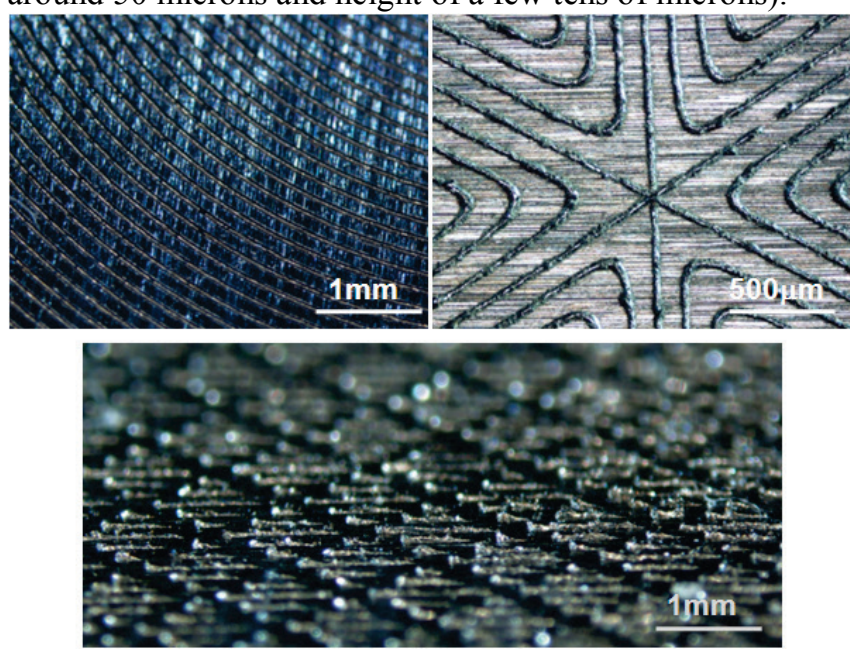

Fig. 9 Complex geometries on tool steel.

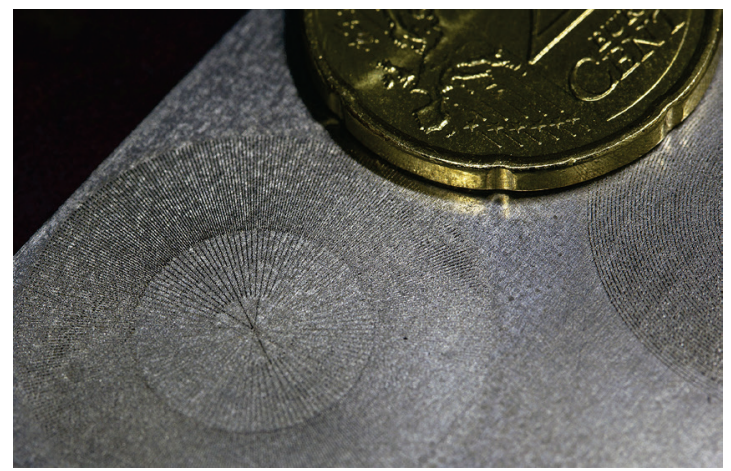

Fig. 10 Complex geometries on titanium alloy.

The homogeneity of those complex geometries was evaluated by SEM micrographic study. Good homogeneity on tracks and precision of features was confirmed.

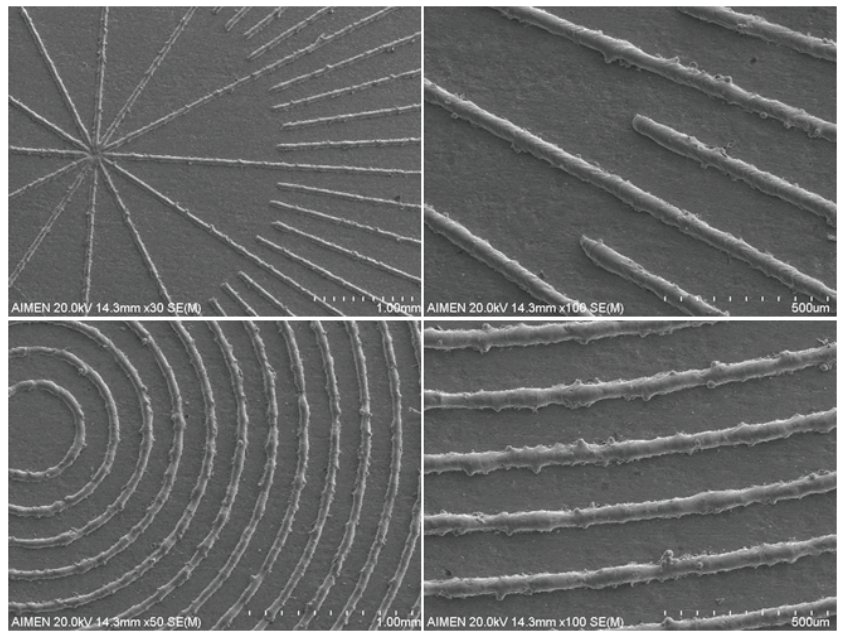

Fig. 11 SEM images of complex geometries on titanium alloy (Radial microtexture and circular geometry).

\subsection{Tribologic behaviour}

The better riblet-like microcladding conditions in terms of penetration and geometrical quality were selected for performing wear tests on tool steel samples.

For assessing the tribological performance of the parent material and cladded tracks, lubricated tribometries were performed by using automotive lubricant oil for pin-on-disc and linear friction tests.

The dynamic counterpart was the cladded sample, while the static counterpart was a cylindrical WC-Co pin. Lineal speed ranged between 0,015 and $0,04 \mathrm{~m} / \mathrm{s}$.

All $\mathrm{L} \mu \mathrm{C}$ microstructured surfaces have a friction coefficient under the value of parent material in as-grinded condition (Ra over 2 microns).

Friction coefficient of the original surface on steel is between 0.15 and 0.2 , while all structured samples have friction coefficient values under 0.15 , being the most important value for the friction performance the spanning between clad tracks.

Better results are obtained for 50 micron spanning $(50 \% \mathrm{FF})$ structures, which even at high loads do not exhibit break of lubricant film at high speeds (up to 600rpm) or with long tests.

Thus, it has been assessed the resistance of the tracks to friction forces (good adhesion to the substrate, good mechanical properties) even at high loads (up to $30 \mathrm{~N}$ on the pin), and the beneficial effect of the structure on the friction forces.

The detrimental effect of vertical dimension inhomogeneities in the tracks is quickly neutralized by deformation of the highest points of each track during the first wearing cycles.

Detected failures in tribometries are not a consequence of the internal defects of the tracks, but result from plastic collapse mechanism of the structure which appears before the track failure (Figure 12). The behaviour is governed by the track geometry instead of the internal defects.

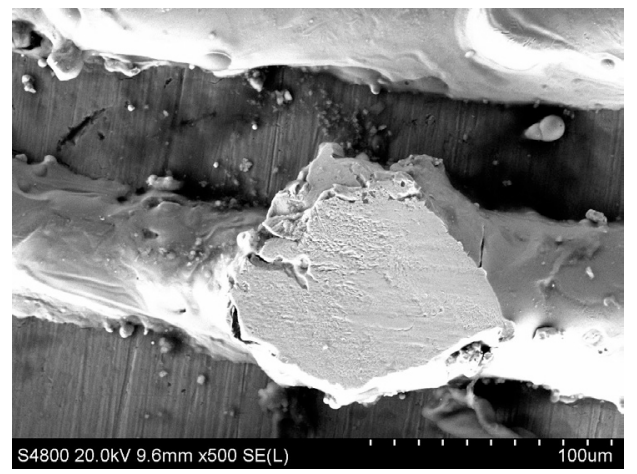

Fig. 12 Deformation of highest points on tool steel track.

Almost no weight loss is detected after long runs of pin-on-disk tests due to this mechanism of flattening followed by progressive reduction of friction coefficient when heights of all tracks are equalized.

Complex geometries generated on titanium alloy samples were studied in terms of wear resistance, obtaining better results than parallel tracks. In this case, selected test was a Flat-on-Disk test (conformal contact, 20 to $50 \mathrm{~N}$ load), in order to confirm the ability of the features to work on conformal configurations. 
In non-lubricated wear tests, the friction coefficient was observed to remain in the same order as the base material, when titanium alloy is used as clad material. Best results were obtained for a radial geometry as shown in Figure 13, where a strong reduction of wearing factor is shown.

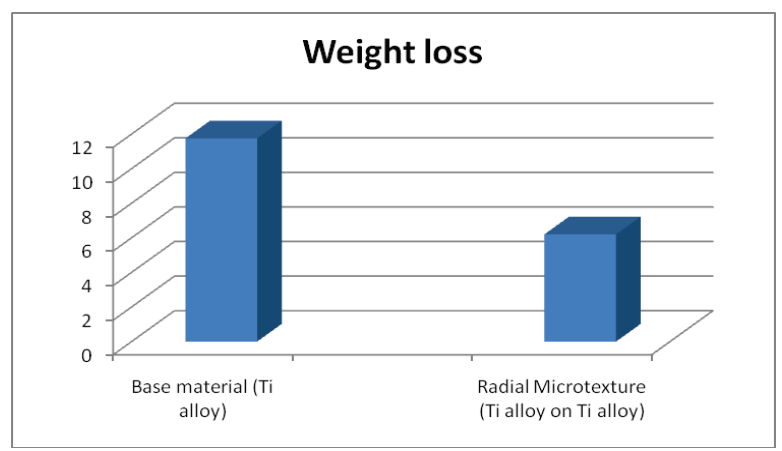

Fig. 13 Weight loss in milligrams.

\section{Conclusions}

Microcladding with powder pre-placing has been tested and demonstrated as a feasible technique for producing micron sized features in different combinations of materials, including titanium alloys.

One important factor to get a good result and repeatable process is the use of a high beam quality laser source with focal spot under 30 microns.

Powder characteristics and placement process highly influence the results. A well tuned pre-placement process will ensure a high quality of the resulting tracks, obtaining homogeneous tracks in width and height, with high dependence on the distribution of particle size and geometry of powder particles. Powders should have monomodal size distribution, with spherical particles and size fewer than 25 microns.

Wet preplacement is preferred to dry process, due to high quality stability and simple application methods. At industrial scale, painting application methods can be used, which are largely optimized and controlled.

The process has shown to be a dependable and affordable solution for the production of microfeatures on large surfaces, thanks to the low power required and the high productivity obtained. Optimal parameters for studied combinations of materials have low laser powder between 20 and $50 \mathrm{~W}$, high scanning speed over $600 \mathrm{~mm} / \mathrm{s}$.

Under these conditions, cladded tracks have good geometrical quality, improved by the use of shielding gas and better wear characteristics than parent material.

Productivity of the $\mathrm{L} \mu \mathrm{C}$ based texturing process was demonstrated. For the creation of riblets with 200 microns pitch it is possible to cover surfaces at rates up to 1 square centimeter per second.

\section{Acknowledgments}

This work is enclosed in the SURFER Project (PID600300-2009-16: Development of laser technology for modification and generation of functional surfaces), supported by national funds from Spanish Government within the call of R\&D\&I Projects for Technological Centers. The authors would like to thank the support of all the consortium members (AIDO, AIMEN, AIN, TEKNIKER).

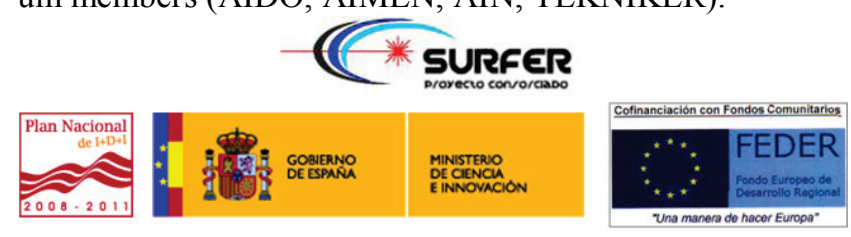

\section{References}

[1] Wan Yi: the effect of laser surface texturing on frictional performance of face seal. Journal of Materials Processing Technology 197, 2008.

[2] Costa, H.L.: Effects of die surface patterning on lubrication in strip drawing. Journal of Materials Processing Technology 209, 2009.

[3] Kovalchenko, Ajayi: The effect of laser surface texturing on transitions in lubrication regimes during unidirectrional sliding contact. Tribology International 38, 2008.

[4] Ren: Micro textures in concentrated-conformal contact lubrication: effect of distribution patterns. Tribology Letters, Oct. 2009.

[5] P. Laakso, S. Ruotsalainen: Sintering of Printed Nanoparticle Structures using Laser Treatment. Icaleo 2009, N205, pages 1360-166 (2009). (Conference proceedings)

[6] J. del Val, R. Comesaña: Laser cladding of Co-based superalloy coatings: Comparative study between Nd:YAG laser and fibre laser. Surface \& Coatings Technology 204 (2010) 1957-1961. (Journal)

[7] D. Geldart.: Types of Gas Fluidization, Powder Technology, 7 (1973) 285-29. (Journal).

[8] R. Chirone, L. Massimilla and S. Russo: Bubble-free fluidization of cohesive powder in an acoustic field. Chemical Engineering Science, 48 (1993) 41-51. (Journal).

[9] I. Yadroitsev, I. Yadroitsava, Ph. Bertrand: Influence of selective laser melting process parameters on morfphology of single tracks from metal powders. LPM2010 Proceedings

(Received: June 02, 2011, Accepted: March 27, 2012) 